\title{
Search for new phases using in-situ reduction technique in the K-Sn-O System
}

Rebecca D. McAuliffe and Daniel P. Shoemaker

Department of Materials Science and Engineering, University of Illinois at Urbana-Champaign

The K-Sn-O system contains several promising phases, such as $\mathrm{K}_{2} \mathrm{Sn}_{3} \mathrm{O}_{7}$, a unique oxide structure that contains large channels with $\mathrm{K}^{+}$ions for possible ion conduction, ${ }^{1}$ and $\mathrm{K}_{2} \mathrm{Sn}_{2} \mathrm{O}_{3}$, a $\mathrm{Sn}^{2+}$-containing compound that has been shown to have electronic and optical properties for transparent conducting oxide applications. $^{2}$ Despite containing these interesting compounds, the K-Sn-O system is a relatively understudied phase space partially due to the difficulty in using many potassium precursors which react in air to form hydrated or hydroxide phases. To search this phase space, we are using an in-situ gas flow setup that we have developed for our laboratory diffractometer. This setup enables us to study the formation of phases during the reduction of $\mathrm{Sn}^{4+}$-containing potassium tin oxide compounds. Reduction reactions can be used to discover new compounds that are difficult to produce using traditional syntheses. This difficulty comes from the use of high temperatures to promote diffusion between precursor materials. By reducing these phases at low temperatures, we can stabilize new phases that are difficult to form using traditional solid-state reactions. This study will not only provide a better understanding of phase formation in the KSn-O system, but also highlight the application of our in-situ gas flow setup that can be used with a laboratory diffractometer.

1. Structural, electronic, and optical properties of $\mathrm{K}_{2} \mathrm{Sn}_{3} \mathrm{O}_{7}$ with an offset hollandite structure. R. D. McAuliffe, C. A. Miller, X. Zhang, B. S. Hulbert, A. Huq, C. dela Cruz, A. Schleife, D. P. Shoemaker, Inorg. Chem. 56 (5) 2914-2918 (2017).

2. Identification and design principles of low hole effective mass p-type transparent conducting oxides. G. Hautier, A. Miglio, G. Ceder, G.-M. Rignanese, and Z. Gonze. Nat. Commun. 4, 2292 (2013). 